Journal of Knot Theory and Its Ramifications

Vol. 18, No. 12 (2009) 1727-1731

(C) World Scientific Publishing Company

\title{
AUTHOR INDEX \\ Volume 18
}

Abe, T.,

An estimation of the alternation number of a torus knot

Adams, C. \& Shayler, T., The projection stick index of knots

Afanasiev, D. M. \& Manturov, V. O.,

On virtual crossing number estimates for virtual links

Afanasiev, D.,

On a generalization of the Alexander polynomial for long virtual knots

Ameur, K. \& Saito, M., Polynomial cocycles of Alexander quandles and applications

Armstrong, J.,

Functors extending the Kauffman bracket

Asami, S. \& Kuga, K., Colorings of torus knots and their twist-spuns by Alexander quandles over finite fields

Baader, S., Cieliebak, K. \& Vogel, T.,

Legendrian ribbons in overtwisted contact structures

Bandieri, P., Cristofori, P. \& Gagliardi, C.,

Non-orientable 3-manifolds admitting colored triangulations with at most 30 tetrahedra

$\mathbf{3}(2009) 363$
$\mathbf{7}(2009) 889$
$\mathbf{1 0}(2009) 757$
$\mathbf{3}(2009) 381$
$\mathbf{9}(2009) 151$
$\mathbf{7}(2009) 985$

Berceanu, B. \& Papadima, Ş.,

Universal representations of braid and braid-permutation groups

Bischof, B., Kogan, R. \& Yetter, D. N.,

On a basis for the framed link vector space spanned by chord diagrams

Blair, R. C., Alternating augmentations of links

Button, J. O.,

Finite covers of the infinite cyclic cover of a knot

Caprau, C., On the $\mathfrak{s l}(2)$ foam cohomology computations

Carter, J. S., Silver, D. S., Williams, S. G., Elhamdadi, M. \& Saito, M., Virtual knot invariants from group biquandles and their cocycles

Cavicchioli, A., Repovš, D. \& Spaggiari, F., Palindrome presentations of rational knots

Chmutov, S., Khoury, M. C. \& Rossi, A.,

Polyak-Viro formulas for coefficients of the Conway polynomial

Cho, J. \& Murakami, J., Some limits of the colored Alexander invariant of the figure-eight knot and the volume of hyperbolic orbifolds
7 (2009) 999

12 (2009) 1663

1 (2009) 67

$1(2009) 75$

9 (2009) 1313

7 (2009) 957

3 (2009) 343

6 (2009) 773

9 (2009) 1271 
Chung, J.-W. \& Lin, X.-S., Torsion of quasi-isomorphisms

Cieliebak, K. See Baader

Cohen, A. M., Gijsbers, D. A. H. \& Wales, D. B., Tangle and Brauer diagram algebras of type $\mathrm{D}_{n}$

Cristofori, P.,

See Bandieri

Degtyarev, A. Oka's conjecture on irreducible plane sextics. II

Deviatov, R., Combinatorial knot invariants that detect Trefoils

Diao, Y., Ernst, C. \& Stasiak, A.,

A partial ordering of knots and links through diagrammatic unknotting

Diao, Y., Hetyei, G. \& Hinson, K.,

Tutte polynomials of tensor products of signed graphs and their applications in knot theory

Dong, F. See Jin

Dubois, J., Huynh, V. \& Yamaguchi, Y., Non-abelian Reidemeister torsion for twist knots

Durumeric, O. C., Local structure of ideal knots, II: Constant curvature case

Dye, H. A. \& Kauffman, L. H.,

Virtual crossing number and the arrow polynomial

Elhamdadi, M., See Carter

Ernst, C., See Diao

Fenn, R.,

Biquandles and their application to virtual knots and links

Friedl, S.,

Realizations of Seifert matrices by hyperbolic knots
9 (2009) 1227

4 (2009) 523

$4(2009) 447$

3 (2009) 381

8 (2009) 1065

9 (2009) 1193

4 (2009) 505

5 (2009) 561

$12(2009) 1711$

3 (2009) 303

$11(2009) 1525$

10 (2009) 1335

7 (2009) 957

4 (2009) 505

6 (2009) 785

11 (2009) 1471
Fukunaga, T.

Homotopy classification of nanophrases in Turaev's theory of words

Gagliardi, C., See Bandieri

Ghuman, S. M., Granda, L. M. \& Tsau, C. M.,

Dehn surgery on singular knots

Gijsbers, D. A. H., See Cohen

Gilmer, P. M., Heegaard genus, cut number, weak $p$-congruence, and quantum invariants

Glasser, D., McDonnell, L., Penderghest, K., Vacha, M. \& Meier, J.,

The geometry of the Dehn complex for a spatial graph

Goda, H., Hayashi, C. \& Song, H.-J.,

Dehn surgeries on 2-bridge links which yield reducible 3-manifolds

Gonçalves, D. L. \& Guaschi, J.,

The lower central and derived series of the braid groups of the finitelypunctured sphere

Goodman, F. M. \& Mosley, H. H.,

Cyclotomic BirmanWenzl-Murakami algebras, I: Freeness and realization as tangle algebras

Granda, L. M. See Ghuman

Green, R. M., On the Markov trace for Temperley-Lieb algebras of type $E_{n}$

Grishanov, S. A., Meshkov, V. R. \& Vassiliev, V. A., Recognizing textile structures by finite type knot invariants

Grishanov, S., See Morton

Guaschi, J., See Gonçalves
7 (2009) 901

3 (2009) 381

4 (2009) 547

4 (2009) 447

10 (2009) 1359

9 (2009) 1175

7 (2009) 917

5 (2009) 651

8 (2009) 1089

4 (2009) 547

2 (2009) 237

2 (2009) 209

12 (2009) 1597

5 (2009) 651 
Hanaki, R.,

Regular projections of

knotted double-handcuff graphs

Hayashi, C., See Goda

Hetyei, G., See Diao

Hinson, K., See Diao

Hoste, J., Torus knots are Fourier$(1,1,2)$ knots

Huynh, V., See Dubois

Ilyutko, D. P. \& Manturov, V. O.,

Introduction to graphlink theory

Jaco, W., Rubinstein, J. H. \& Sedgwick, E.,

Finding planar surfaces in knot- and link-manifolds

Jin, X., Dong, F. \& Tay, E. G.,

Determining the component number of links corresponding to lattices

Juyumaya, J. Lambropoulou, S.,

An invariant for singular knots

Kalfagianni, E., A note on quantum 3manifold invariants and hyperbolic volume

Kauffman, L. H., See Dye

Kauffman, L. H., An extended bracket polynomial for virtual knots and links

Khoury, M. C., See Chmutov

Kim, S.-G., Alexander polynomials and orders of homology groups of branched covers of knots

Kogan, R. See Bischof

Kuga, K., See Asami
11 (2009) 1475

7 (2009) 917

5 (2009) 561

5 (2009) 561

2 (2009) 265

3 (2009) 303

6 (2009) 791

3 (2009) 397

12 (2009) 1711

6 (2009) 825

1 (2009) 33

10 (2009) 1335

10 (2009) 1369

6 (2009) 773

7 (2009) 973

12 (2009) 1663

9 (2009) 1259
Kuppum, S. \& Zhang, X., Roots of unity associated to strongly detected boundary slopes

Lambropoulou, S., See Juyumaya

Lauda, A. D. \& Pfeiffer, H., Open-closed TQFTs extend Khovanov homology from links to tangles

Lee, S. Y. \& Seo, M., Formulas for the Casson invariant of certain integral homology 3-spheres

Lee, S.,

Toroidal surgeries on genus two knots

Lei, F., See Yin

Li, Y. 2-string free tangles and incompressible surfaces

Lin, X.-S., See Chung

Liu, X., The quantum Teichmüller space as a noncommutative algebraic object

Madeti, P. \& Mishra, R., Minimal degree sequence for torus knots of type $(p, q)$

Madras, N., Sumners, D. W. \& Whittington, S. G., Almost unknotted embeddings of graphs in $Z^{3}$ and higher dimensional analogues

Mann, C., McLoud-Mann, J., Ranalli, R., Smith, N. \& McCarty, B.,

Minimal knotting numbers

Manturov, V. O., See Afanasiev

Manturov, V. O., See Ilyutko

Margalit, D. \& McCammond, J.,

Geometric presentations for the pure braid group

Matveev, S. \& Polyak, M., A simple formula for the Casson-Walker invariant
12 (2009) 1623

6 (2009) 825

1 (2009) 87

11 (2009) 1551

9 (2009) 1205

2 (2009) 199

8 (2009) 1081

9 (2009) 1227

5 (2009) 705

4 (2009) 485

8 (2009) 1031

8 (2009) 1159

6 (2009) 757

6 (2009) 791

1 (2009) 1

6 (2009) 841 
McCammond, J., See Margalit

McCarty, B., See Mann

McDonnell, L., See Glasser

McLoud-Mann, J., See Mann

Meier, J., See Glasser

Meshkov, V. R., See Grishanov

Mishra, R., See Madeti

Miyazawa, Y., A multi-variable polynomial invariant for unoriented virtual knots and links

Miyazawa, Y.,

A virtual link polynomial and the virtual crossing number

Mohamad, A. \& Yashiro, T.,

On triple point numbers of 5-colorable 2-knots

Moriuchi, H., An enumeration of thetacurves with up to seven crossings

Morton, H. R. \& Grishanov, S., Doubly periodic textile structures

Morton, H. R. \& Ryder, N., Invariants of genus 2 mutants

Mosley, H. H., See Goodman

Murakami, J., See Cho

Nakanishi, Y., Shibuya, T. \& Tsukamoto, T., Free self delta-triviality of delta-split links

Ni, Y., Closed 3-braids are nearly fibred

Nouh, M. A.,

Genera and degrees of torus knots in $\mathbb{C} P^{2}$

$$
\begin{aligned}
& \mathbf{1}(2009) 1 \\
& \mathbf{8}(2009) 1159 \\
& \mathbf{9}(2009) 1175 \\
& \mathbf{8}(2009) 1159 \\
& \mathbf{9}(2009) 1175 \\
& \mathbf{2}(2009) 209 \\
& \mathbf{4}(2009) 485 \\
& \mathbf{5}(2009) 625
\end{aligned}
$$$$
5 \text { (2009) } 605
$$$$
11 \text { (2009) } 1493
$$$$
2 \text { (2009) } 167
$$$$
12 \text { (2009) } 1597
$$$$
10 \text { (2009) } 1423
$$$$
8 \text { (2009) } 1089
$$$$
9 \text { (2009) } 1271
$$$$
11 \text { (2009) } 1539
$$$$
12 \text { (2009) } 1637
$$

9 (2009) 1299
Ogasa, E.,

Local move identities for the Alexander polynomials of high-dimensional knots and inertia groups

Oikawa, T., On a local move for virtual knots and links

Pakovich, F.,

Solution of the Hurwitz problem for Laurent polynomials

Papadima, Ş. See Berceanu

Penderghest, K., See Glasser

Pervova, E. \& Petronio, C., Complexity of links in 3manifolds

Petronio, C., See Pervova

Pfeiffer, H., See Lauda

Polyak, M., See Matveev

Ranalli, R., See Mann

Repovš, D., See Cavicchioli

Rossi, A., See Chmutov

Rubinstein, J. H., See Jaco

Ryder, N., See Morton

Saeki, O. \& Takeda, Y., Surface links and their generic planar projections

Saito, M., See Ameur

Saito, M., See Carter

Sayari, N. Reducible Dehn surgery and the bridge number of a knot

Sedgwick, E. See Jaco

Seo, M. See Lee

Shayler, T., See Adams
4 (2009) 531

11 (2009) 1577

2 (2009) 271

7 (2009) 999

9 (2009) 1175

10 (2009) 1439

10 (2009) 1439

1 (2009) 87

6 (2009) 841

8 (2009) 1159

3 (2009) 343

6 (2009) 773

3 (2009) 397

$10(2009) 1423$

1 (2009) 41

2 (2009) 151

7 (2009) 957

4 (2009) 493

3 (2009) 397

11 (2009) 1551

7 (2009) 889 
Shibuya, T., See Nakanishi

Silver, D. S., See Carter

Smith, N., See Mann

Song, H.-J., See Goda

Spaggiari, F., See Cavicchioli

Stasiak, A., See Diao

Sumners, D. W., See Madras

Takata, T., Ohtsuki invariants for integral homology spheres and Habiro's cyclotomic expansion

Takeda, Y., See Saeki

Tang, J., See Yin

Taniyama, K., Unknotting numbers of diagrams of a given nontrivial knot are unbounded

Tay, E. G., See Jin

Todd, R. G., Khovanov homology and the twist number of alternating knots

Traldi, L. \& Zulli, L., A bracket polynomial for graphs, I

Tsau, C. M., See Ghuman

Tsukamoto, T., See Nakanishi

Tuffley, C., Generalized knot groups distinguish the square and Granny knots (with an appendix by David Savitt)

Vacha, M., See Glasser

$\mathbf{1 1}(2009) 1539$
$\mathbf{7}(2009) 957$
$\mathbf{8}(2009) 1159$
$\mathbf{7}(2009) 917$
$\mathbf{3}(2009) 343$
$\mathbf{4}(2009) 505$
$\mathbf{8}(2009) 1031$
$\mathbf{1}(2009) 21$
$\mathbf{9}(2009) 1175$
$\mathbf{1}(2009) 41$
$\mathbf{2}(2009) 199$
$\mathbf{8}(2009) 1049$
$\mathbf{8}(2009) 1129$
$\mathbf{1 2}(2009) 1681$
$\mathbf{1 2}(2009) 1711$

Vassiliev, V. A., See Grishanov

Viro, J., Lines joining components of a link

Viro, O., Twisted acyclicity of a circle and signatures of a link

Vogel, T., See Baader

Wales, D. B., See Cohen

Whittington, S. G., See Madras

Widmer, T., Quasi-alternating montesinos links

Williams, S. G., See Carter

Winter, B., The classification of spun torus knots

Yaguchi, Y., Isotropy subgroup of Hurwitz action of the 3-braid group on the braid systems

Yamaguchi, Y., See Dubois

Yashiro, T., See Mohamad

Yasuda, T., Ribbon 2-knots with distinct ribbon types

Yetter, D. N., See Bischof

Yin, X., Tang, J. \& Lei, F., On maximal collections of essential annuli in a handlebody II

Zhang, Q., Boundary slopes of immersed surfaces in Haken manifolds

Zhang, X., See Kuppum

Zulli, L., See Traldi
2 (2009) 209

6 (2009) 865

6 (2009) 729

4 (2009) 523

$4(2009) 447$

8 (2009) 1031

10 (2009) 1459

7 (2009) 957

9 (2009) 1287

7 (2009) 1021

3 (2009) 303

11 (2009) 1493

11 (2009) 1509

$12(2009) 1663$

2 (2009) 199

5 (2009) 591

12 (2009) 1623

12 (2009) 1681 